Ingeniería

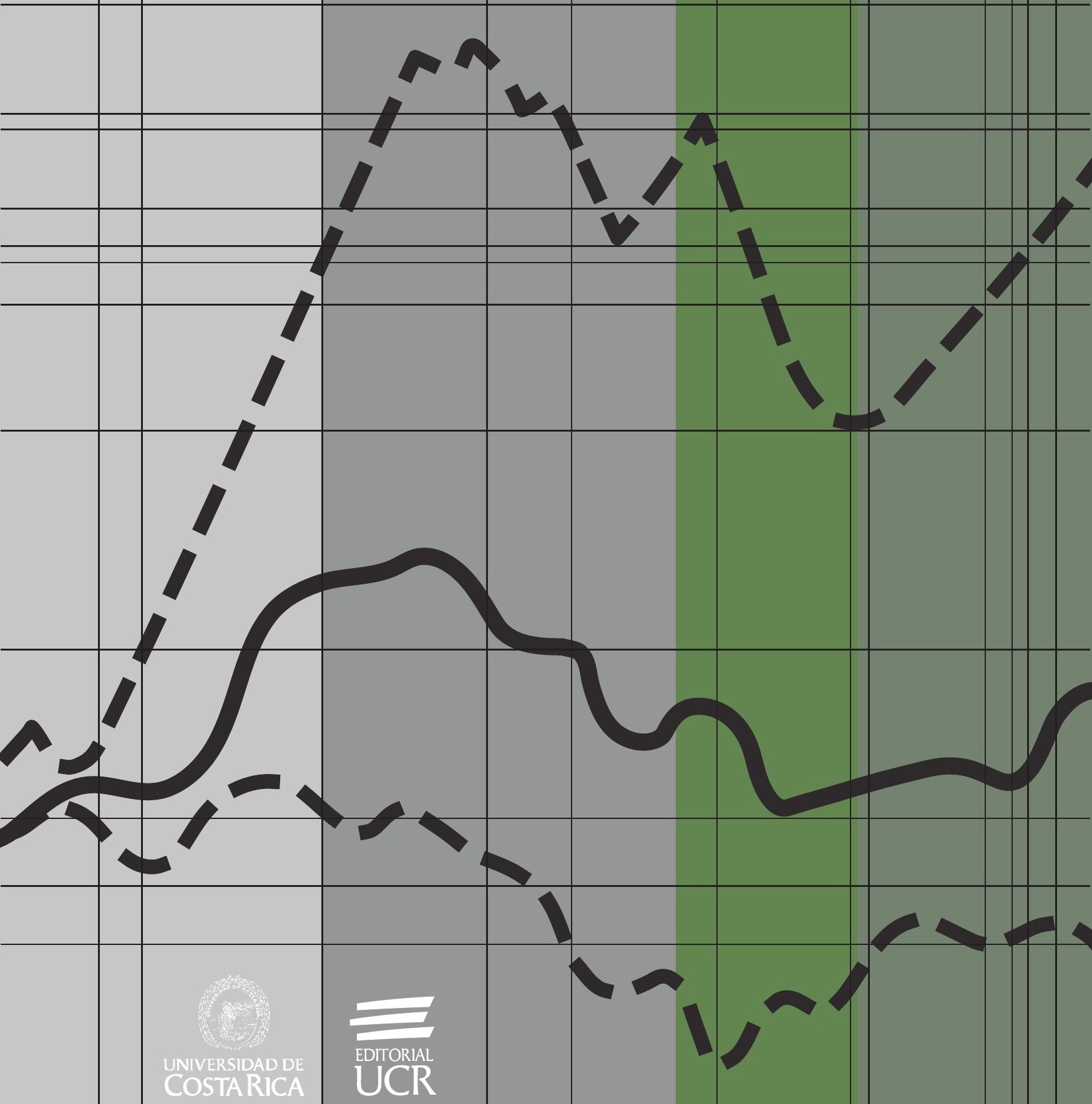




\title{
Efecto de sales de boro en la densidad y en el módulo de elasticidad de tres maderas mexicanas
}

\author{
Effect of Boron Salts on the Density and on the Modulus of \\ Elasticity of Three Mexican Woods
}

\author{
Javier Ramón Sotomayor Castellanos \\ Universidad Michoacana de San Nicolás de Hidalgo, México \\ madera999@yahoo.com \\ María del Pilar Giraldo Forero \\ Centre Tecnològic Forestal de Catalunya, España \\ pilar.giraldo@incafust.cat \\ Jordi Gené Sera \\ Centre Tecnològic Forestal de Catalunya, España \\ jordi.gene@incafust.cat \\ Eduard Correal Mòdol \\ Centre Tecnològic Forestal de Catalunya, España \\ eduard.correal@incafust.cat \\ Marcel Vilches Casals \\ Centre Tecnològic Forestal de Catalunya, España \\ marcel.vilches@fbs.cat
}

Recibido: 11 de diciembre 2017

Aceptado: 21 de marzo 2018

\section{Resumen}

Las especies Guazuma ulmifolia, Spathodea campanulata y Abies religiosa tienen potencial para la fabricación de productos de madera con valor agregado. Sin embargo, no existe información sobre sus propiedades de retención de substancias protectoras, ni de su posible efecto sobre la densidad y el módulo de elasticidad. El objetivo de la investigación fue analizar el efecto del tratamiento con baño caliente-frío de sales de boro sobre la densidad y el módulo de elasticidad en muestras de madera de G. ulmifolia, S. campanulata y A. religiosa. Antes y después del tratamiento se determinaron la densidad de la madera, el módulo de elasticidad en flexión estática y la retención de sales de boro. Se realizaron pruebas de diferencias de medias y después del tratamiento se efectuó entre las tres especies un análisis de varianza de la variable retención. En las muestras de madera de G. ulmifolia, S. campanulata y A. religiosa la retención de sales de boro fue diferente para cada una de ellas. La densidad después de aplicar las sales aumentó 
en G. ulmifolia y A.religiosa y disminuyó en S. campanulata. Las sales de boro no modificaron el módulo de elasticidad de la madera de las tres especies.

\title{
Palabras clave:
}

Guazuma ulmifolia, Spathodea campanulata, Abies religiosa, flexión estática, retención de sales de boro.

\begin{abstract}
The species Guazuma ulmifolia, Spathodea campanulata and Abies religiosa have potential for the fabrication of wood products with benefit. However, there is no information about its properties regarding the retention of protection substances, neither of its possible effect on the wood density and the modulus of elasticity. The research aimed to analyze, in samples of $G$. ulmifolia, S. campanulata and A. religiosa wood, the effect of the hot-cold bath of the boron salts on the density and the modulus of elasticity. Before and after the treatment, the wood density, the modulus of elasticity, and the retention of boron salts were established. Differences-of-means tests between the species were performed, and after the treatment an analysis of variance was carried for the variable retention. In the specimens of G. ulmifolia, S. campanulata y A.religiosa wood, the retention of boron salts was different for each one of them. After the application of the salts, the density of G. ulmifolia y A.religiosa increased, and decreased in S. campanulata. The boron salts did not modify the modulus of elasticity of the wood for the three species.
\end{abstract}

Keywords:

Guazuma ulmifolia, Spathodea campanulata, Abies religiosa, static bending, retention of boron salts. 


\section{INTRODUCCIÓN}

La madera es un componente constructivo que se complementa con otros materiales y tecnologías propias de la industria de la construcción (Walker, 2006). La madera debe mantenerse en ciertas condiciones para evitar daños por agentes biológicos y factores físicos que modifican su composición y provocan su degradación (Bettiol, Ceccato, Pigouni, Modena y Simonini, 2016). En México existen especies maderables abundantes y demanda de madera que puede emplearse como material para la construcción (Ávila, Herrera y Raya, 2012). No obstante, en la literatura mexicana especializada son escasos los parámetros derivados de investigaciones orientadas a la industria de la madera, y a la protección de madera con substancias preventivas del deterioro, como sales de boro.

Las sales de boro son una sustancia protectora para la madera con un amplio espectro en su acción fungicida e insecticida y de poca toxicidad (Lloyd, Fogel y Vizel, 2001; Temiz, Alfredsen, Eikenes y Terz1ev, 2008). Su aplicación es óptima en elementos de madera sin contacto con el suelo y la solubilidad de los compuestos de boro le permite preservar especies de madera que son difíciles de tratar con preservantes a base de cobre y zinc (Obanda, Shupe y Barnes, 2008). Además, sus propiedades de difusión al interior del plano leñoso le facilitan su distribución uniforme cuando la madera tiene un alto contenido de humedad. Así que, por ser inodoras, incoloras y no inflamables, la aplicación de sales de boro en la madera es una de las estrategias más efectivas de preservación (Tsunoda, 2001; Tondi et al., 2012).

$\mathrm{Al}$ interior de la madera, la presencia de agua es el principal factor responsable de la migración del boro. De tal forma, una vez en servicio las sales de boro se disuelven en contacto con el agua, proceso llamado lixiviado (Baysal, Sonmez, Colak y Toker 2006; Caldeira, 2010). Con el objeto de impedir la movilidad del preservante por el efecto del lixiviado, se han propuesto tratamientos térmicos para reducir su higroscopicidad, procesos de densificando de la madera para reducir los espacios vacíos en el tejido leñoso, así como técnicas de impregnación con vapor (Salman et al., 2014; Obounou-Akong, Gérardin, Thévenon y Gérardin-Charbonnier, 2015).

La cantidad de substancia preservante que una pieza de madera puede retener depende de su capacidad de absorción de fluidos y, debido a la variabilidad en su estructura anatómica, cada especie tiene un comportamiento distinto de acuerdo con el tratamiento aplicado (Ávila et al., 2012). Cuantitativamente, la retención de sales de boro es función también de la concentración de la substancia preservadora empleada en el tratamiento (Simsek, Baysal y Peker, 2010).

Berrocal, Muñoz y González (2004) aplican sales de boro en probetas de Gmelina arborea, con dimensiones de $0,05 \mathrm{~m} \times 0,025 \mathrm{~m} \times 0,5 \mathrm{~m}, \mathrm{y}$ logran retenciones de $0,32 \mathrm{~kg} \mathrm{~m}^{-3}$ a $1,28 \mathrm{~kg} \mathrm{~m}^{-3}$, con un método de inmersión-difusión y una concentración de sales de boro de $12 \%$. Dhamodaran y Gnanaharan (2007) obtienen una retención de $3,70 \mathrm{~kg} \mathrm{~m}^{-3}$ a $11,60 \mathrm{~kg} \mathrm{~m}^{-3}$, en piezas de madera de Eucalyptus grandis, aplicando sales de boro y empleando un método de presión con una concentración de $6 \%$. Simsek et al. (2010), empleando probetas de $0,02 \mathrm{~m} \times 0,02 \mathrm{~m} \times 0,36 \mathrm{~m}$, con un proceso al vacío 
con concentraciones de sales de boro de $0,25 \%$ a $3 \%$, reportan retenciones de 17,42 $\mathrm{kg} \mathrm{m}^{-3}$ para madera de Fagus orientalis y de $15,98 \mathrm{~kg} \mathrm{~m}^{-3}$ para la de Pinus sylvestris.

Tondi et al. (2012), impregnaron con sales de boro al $6 \%$ en solución acuosa probetas de $0,025 \mathrm{~m} \times 0,015 \mathrm{~m} \times 0,05 \mathrm{~m}$ con un proceso de preservación diseñado específicamente. Los investigadores encontraron retenciones de $2,88 \mathrm{~kg} \mathrm{~m}^{-3}$ a $9,16 \mathrm{~kg} \mathrm{~m}^{-3}$ para madera de Fagus sylvatica y de $3,25 \mathrm{~kg} \mathrm{~m}^{-3}$ a $9,57 \mathrm{~kg} \mathrm{~m}^{-3}$ para la de Pinus Sylvestris. Salman et al. (2014), preservaron con sales de boro al $4 \%$ probetas de 0,015 $\mathrm{m} \times 0,005$ $\mathrm{m} \times 0,050 \mathrm{~m}$ y lograron una retención de $4,2 \mathrm{~kg} \mathrm{~m}^{-3}$ para madera de Pinus sylvestris y de $5,7 \mathrm{~kg} \mathrm{~m}^{-3}$ para la de Fagus sylvatica.

Berrocal et al. (2004), Dhamodaran y Gnanaharan (2007), Simsek et al. (2010) y Tondi et al. (2012) emplean diferentes especies de madera, diversas concentraciones de sales de boro y variados métodos de aplicación. De tal forma, los resultados son difíciles de comparar. No obstante, estos autores han verificado la capacidad de retención de sales de boro en maderas con muy distintas estructuras anatómicas y con diversas condiciones de tiempo, presión y temperatura en los tratamientos de impregnado.

El tratamiento de baño caliente-frío aprovecha la diferencia de presión ocasionada por la exposición de la madera a un medio acuoso con temperatura de $60{ }^{\circ} \mathrm{C}$, de tal forma que el cambio brusco de temperatura por la inmersión de la madera caliente en una solución de sales a temperatura de $20{ }^{\circ} \mathrm{C}$ facilita la difusión de las sales al interior del plano leñoso (Walker, 2006). Así, se logran retenciones mayores a las sugeridas por la Asociación Americana de Preservadores de Madera, la cual recomienda una retención mínima de sales de boro de $2,72 \mathrm{~kg} \mathrm{~m}^{-3}$ en la madera para elementos estructurales empleados en interiores (American Wood-Preservers' Association, 2014).

Las especies Guazuma ulmifolia Lam., Spathodea campanulata Buch.-Ham. ex DC. y Abies religiosa (Kunth) Schltdl. et Cham. son endémicas de México y presentan potencial para la fabricación de productos de alto valor agregado. La información sobre sus características físicas y mecánicas está reportada por Sotomayor (2015). Sin embargo, no existe información sobre sus propiedades de retención de substancias protectoras, ni de su posible efecto sobre el módulo de elasticidad.

Trabajos sobre la aptitud para la preservación de maderas mexicanas recomiendan clasificaciones de acuerdo a la facilidad y/o a la dificultad de la madera para su preservado. Algunas de ellas se basan en trabajos relevantes en su momento. Pero, dado el desarrollo actual de productos para la protección de la madera, se pueden considerar como desactualizados (Ávila et al., 2012). Estos autores reportan trabajos sobre la aptitud para la preservación de maderas mexicanas en los que el proceso de preservación "baño caliente-frío" ha sido empleado con éxito. Esta técnica solo necesita instrumentos accesibles y resulta una técnica económica.

En la literatura especializada mexicana existe información de las características de las especies en estudio (Sotomayor, 2015) y sobre la experimentación con sales de boro en la madera y sus estrategias de aplicación (Cruz, 2010; Ávila et al., 2012). Sin embargo, no se encontraron trabajos publicados que integren pruebas de preservado con sales de boro de madera y su efecto sobre el módulo de elasticidad. 
El objetivo de la investigación fue establecer el efecto del tratamiento de impregnación de la madera de G. ulmifolia, S. campanulata y A. religiosa en sus propiedades de densidad y módulo de elasticidad.

\section{MATERIALES Y MÉTODOS}

\section{Materiales}

Madera de G. ulmifolia, S. campanulata y A.religiosa se recolectó en aserraderos del Estado de Michoacán (Longitud oeste 101 49’ 59,99”, Latitud Norte 19 10’0,01”), México. La madera se almacenó durante 24 meses en una cámara de acondicionamiento marca FITECMA, con una temperatura de $20^{\circ} \mathrm{C}\left( \pm 1{ }^{\circ} \mathrm{C}\right)$ y una humedad relativa del aire de $65 \%( \pm 2 \%)$ hasta que el peso de la madera fue constante. Para las pruebas de flexión estática, y para cada una de las especies, se prepararon lotes de 35 probetas con dimensiones de $0,02 \mathrm{~m} \times 0,02 \mathrm{~m} \times 0,32 \mathrm{~m}$, respectivamente en las direcciones radial, tangencial y longitudinal de la madera.

Para la determinación del contenido de humedad de la madera (International Organization for Standardization, 2014a) y de su densidad (International Organization for Standardization, 2014b) y de su se prepararon tres lotes adicionales de 35 probetas correspondientes a cada una de las especies, con dimensiones de 0,02 $\mathrm{m} \times 0,02 \mathrm{~m} \times$ $0,06 \mathrm{~m}$. Estas probetas tuvieron características similares y provenían del mismo lote de madera que se destinó al tratamiento. La madera no contenía anomalías estructurales ni defectos de crecimiento. Para fines de comparación, se consideró que la humedad fue constante y, en consecuencia, este factor no influyó en los resultados.

Se realizó una primera serie de pruebas de flexión estática a cuyos resultados se les identificó como "antes tratamiento" (at). Una vez terminado el tratamiento de baño caliente-frío se efectuaron las mediciones pertinentes. En seguida, las probetas se almacenaron por tres meses en las mismas condiciones en que se estabilizó la madera antes del tratamiento (temperatura de $20{ }^{\circ} \mathrm{C}$ y humedad relativa del aire de $65 \%$ ) hasta que alcanzaron un peso constante. Posteriormente, se realizó la segunda serie de pruebas de flexión estática a cuyos resultados se les identificó como "después tratamiento" (dt).

\section{Tratamiento de impregnación}

Se prepararon 30 L de solución de trihidróxido de boro (Ácido bórico, 39,4\%) y tetraborato de sodio (borato de sodio, 60,6 \%) con una concentración al 3\%, de acuerdo con la norma NMX-C-410-ONNCCE-1999 (Organismo Nacional de Normalización y Certificación de la Construcción y Edificación, 1999). Las probetas se impregnaron con el método "baño caliente-frío" siguiendo el protocolo propuesto por Ávila et al. (2012). La madera se sumergió durante 8 h en un baño de agua con temperatura de $60{ }^{\circ} \mathrm{C}$ y presión atmosférica. A continuación, las probetas se sumergieron durante 16 $\mathrm{h}$ en un baño frío, con la solución de sales de boro, y temperatura de $23{ }^{\circ} \mathrm{C}$ y presión 
atmosférica. Posteriormente, se determinó la absorción, parámetro necesario para calcular después la retención de las sales en cada probeta.

La absorción se determinó de acuerdo con Ávila et al. (2012), con la fórmula (1):

$$
\mathrm{A}=\frac{\mathrm{P}_{\mathrm{f}}-\mathrm{P}_{\mathrm{i}}}{\mathrm{V}_{\mathrm{dt}}}
$$

Donde:
A: $\quad$ Absorción $\left(\mathrm{kg} \mathrm{m}^{-3}\right)$
$\mathrm{P}_{\mathrm{i}}$ : $\quad$ Peso inicial de la probeta antes tratamiento $(\mathrm{kg})$
$\mathrm{P}_{\mathrm{f}}: \quad \quad$ Peso final de la probeta después tratamiento $(\mathrm{kg})$
$\mathrm{V}_{\mathrm{dt}}: \quad$ Volumen de la probeta después tratamiento $\left(\mathrm{m}^{3}\right)$

La retención se determinó de acuerdo con Simsek, Baysal, Yilmaz y Culha (2013), con la fórmula (2):

$$
\mathrm{R}=\frac{\mathrm{A} \times \mathrm{C}}{100}
$$

Donde:
R: $\quad$ Retención $\left(\mathrm{kg} \mathrm{m}^{-3}\right)$
A: $\quad$ Absorción $\left(\mathrm{kg} \mathrm{m}^{-3}\right)$
C: $\quad$ Concentración (\%)

\section{Pruebas de flexión estática}

Las pruebas de flexión estática consistieron en cargar las probetas en medio de su portada de flexión con una velocidad de $2 \mathrm{~mm} / \mathrm{min}$ en el intervalo elástico de 300 $\mathrm{N}$ a $600 \mathrm{~N}$, correspondiente al $20 \%$ del dominio lineal de la relación carga-deformación. Las pruebas fueron realizadas en una maquina universal de ensayos mecánicos de $30 \mathrm{KN}$ de capacidad. El límite elástico fue estimado previamente en tres probetas adicionales y cargadas hasta la ruptura. La figura 1 presenta la configuración de las pruebas de flexión y la figura 2 presenta una fotografía de una prueba de flexión estática. 


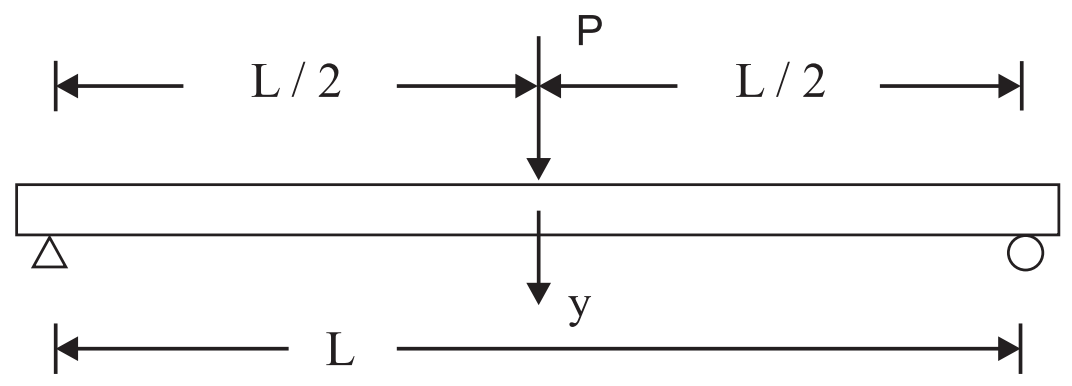

Figura 1. Configuración de las pruebas de flexión estática. $\mathrm{P}=$ carga; $\mathrm{y}=$ deformación; $\mathrm{L}=$ distancia entre apoyos $(0,3 \mathrm{~m})$.

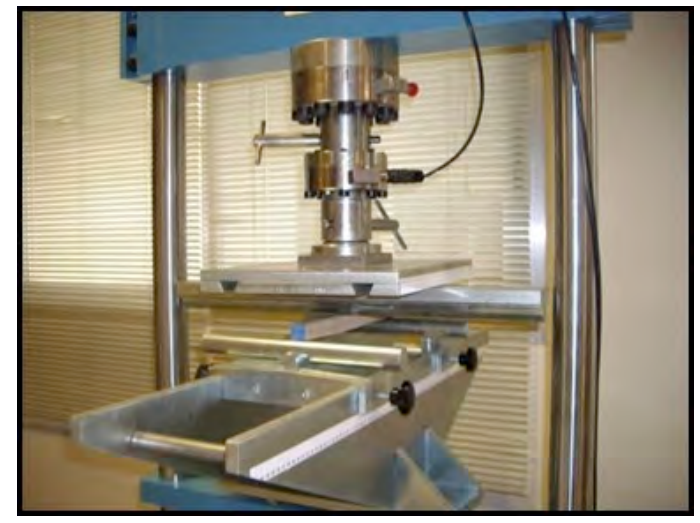

Figura 2. Prueba de flexión estática.

El módulo de elasticidad se calculó de acuerdo con Koman, Feher, Abraham y Taschner (2013), empleando la fórmula (3):

$$
\mathrm{MOE}=\frac{\mathrm{P}}{\mathrm{y}} \frac{\mathrm{L}^{3}}{48 \mathrm{I}}
$$

Donde:

MOE: Módulo de elasticidad $\left(\mathrm{N} \mathrm{m}^{-2}\right)$

P: $\quad$ Carga $(\mathrm{N})$

L: $\quad$ Distancia entre apoyos $(\mathrm{m})$

y: Deformación en L/2 (m)

I: $\quad$ Momento de inercia de la sección trasversal $\left(\mathrm{m}^{4}\right)$ 


\section{Diseño experimental}

Para las tres especies, G. ulmifolia, S. campanulata y A. religiosa, las variables de respuesta fueron: la densidad y el módulo de elasticidad, ambos parámetros para "antes tratamiento" y "después tratamiento". La retención de sales de boro se consideró únicamente como variable después del tratamiento. El tratamiento de impregnación se consideró el factor de variación. Para las variables, se calcularon la media, la desviación estándar y el coeficiente de variación.

Tres experimentos se diseñaron. El primero consistió en pruebas de normalidad, calculando el apuntalamiento y el sesgo, para confirmar que los datos de las muestras provenían de distribuciones normales. Cuando la prueba de normalidad verificó que los datos provenían de distribuciones normales, se realizó, para la densidad y el módulo de elasticidad, el segundo experimento que consistió en pruebas $t$ de Student de diferencias de medias para un nivel de confianza de $95 \%$. La hipótesis nula $\mathrm{H}_{0}: \overline{\mathrm{x}}_{1}-\overline{\mathrm{x}}_{2}=0$ (P $\leq 0,05)$ se contrastó con la hipótesis alterna $\mathrm{H}_{\mathrm{A}}: \overline{\mathrm{x}}_{1}-\overline{\mathrm{x}}_{2} \neq 0(\mathrm{P} \leq 0,05)$. Los subíndices 1 y 2 representan el valor de las variables de respuesta "antes tratamiento" y "después tratamiento". El tercer experimento consistió en un análisis de varianza de la variable retención entre las tres especies. Los cálculos estadísticos fueron realizados con el programa Statgraphics ${ }^{\circledR}$.

\section{RESULTADOS Y DISCUSIÓN}

Los valores de sesgo y de apuntalamiento de la densidad (de los módulos de elasticidad y de la retención) se posicionaron en el intervalo $-2 \mathrm{a}+2$. Como resultado, se admitió que las muestras provinieron de distribuciones normales.

Para las variables densidad "antes tratamiento" y "después tratamiento" de $G$. ulmifolia el valor de $\mathrm{P}$ fue mayor que el valor límite de $0,05(\mathrm{P}=0,0749, \alpha \leq 0,05)$. De tal forma, que no se pudo rechazar la hipótesis nula en favor de la alternativa. En contraste, para las variables densidad "antes tratamiento" y "después tratamiento" de S.campanulata $(\mathrm{P}=0,0025, \alpha \leq 0,05)$ y de A. religiosa $(\mathrm{P}=0,0399, \alpha \leq 0,05)$ los valores de $\mathrm{P}$ fueron menores que el valor límite. Así, se rechazó la hipótesis nula en favor de la alternativa. Para las variables módulo de elasticidad "antes tratamiento" y "después tratamiento" los valores de $\mathrm{P}$ fueron mayores que el valor límite: G. ulmifolia (P $=0,1984, \alpha \leq 0,05), S$. campanulata $(\mathrm{P}=0,0763, \alpha \leq 0,05)$ y A. religiosa $(\mathrm{P}=0,7750$, $\alpha \leq 0,05)$. De tal forma, que no se rechazó la hipótesis nula en favor de la alternativa.

Para la variable retención el valor de $\mathrm{P}$ fue menor que el valor límite $(\mathrm{P}<0,001$, $\alpha \leq 0,05)$, en consecuencia, se deduce que existió una diferencia significativa entre las medias de la retención para G. ulmifolia, S. campanulata y A. religiosa. De acuerdo con Zauer, Pfriem, Mechtcherine y Wagenführ (2013), la retención de una madera depende en gran medida de su estructura anatómica. El tipo de porosidad y la proporción de tejidos que conforman una capa de crecimiento son factores propios del plano leñoso particular a cada una de las maderas estudiadas. De tal forma que las diferencias en su estructura 
anatómica son factores que influyen en su capacidad para absorber sales de boro y en consecuencia, su retención (Zauer, Hempel, Pfriem, Mechtcherine y Wagenführ, 2014).

El contenido de humedad en la madera antes y después del tratamiento varió entre $10 \%$ y $12 \%$ para las tres especies y presentó coeficientes de variación entre 2,7 \% y $3,7 \%$. La densidad antes tratamiento de G. ulmifolia se clasifica como Alta, y las de S. campanulata y A. religiosa como muy bajas de acuerdo a la clasificación propuesta por Sotomayor y Ramírez (2013). La densidad después tratamiento, comparativamente con la densidad antes tratamiento, aumentó un 2,6 \% para G. ulmifolia y un 3,6 \% para A. religiosa. En contraste, la densidad después tratamiento de $S$. campanulata disminuyó un 5,9\%.

Los módulos de elasticidad antes tratamiento de G. ulmifolia y de A. religiosa fueron menores en relación a los reportados por Sotomayor (2015). Para G. ulmifolia, con densidad de $360 \mathrm{~kg} \mathrm{~m}^{-3}$, la reducción fue del $19,7 \%$ y para A. religiosa, con densidad de $380 \mathrm{~kg} \mathrm{~m}^{-3}$, la disminución fue del 34,5\% (tabla 1).

Tabla 1. Densidad, módulo de elasticidad y retención de sales de boro en madera de G. ulmifolia, $S$. campanulata y A. religiosa, antes y después tratamiento.

\begin{tabular}{|c|c|c|c|c|c|}
\hline & \multicolumn{2}{|c|}{ Antes tratamiento } & \multicolumn{3}{|c|}{ Después tratamiento } \\
\hline & $\varrho_{\mathrm{CH}}$ & MOE & $\varrho_{\mathrm{CH}}$ & MOE & $\mathrm{R}$ \\
\hline \multicolumn{6}{|c|}{ G. ulmifolia } \\
\hline$\overline{\mathrm{x}}$ & 730 & 8226 & 749 & 8537 & 9,70 \\
\hline$\sigma$ & 46 & 1027 & 42 & 975 & 0,40 \\
\hline $\mathrm{CV}(\%)$ & 6,4 & 12,5 & 5,6 & 11,4 & 4,2 \\
\hline \multicolumn{6}{|c|}{ S. campanulata } \\
\hline$\overline{\mathrm{x}}$ & 357 & 3563 & 336 & 3846 & 14,31 \\
\hline$\sigma$ & 30 & 657 & 26 & 661 & 0,60 \\
\hline $\mathrm{CV}(\%)$ & 8,5 & 18,6 & 7,6 & 17,2 & 4,2 \\
\hline \multicolumn{6}{|c|}{ A. religiosa } \\
\hline$\overline{\mathrm{x}}$ & 419 & 10653 & 434 & 10575 & 7,23 \\
\hline$\sigma$ & 29 & 1161 & 28 & 1118 & 1,26 \\
\hline $\mathrm{CV}(\%)$ & 6,8 & 10,9 & 6,4 & 10,6 & 17,4 \\
\hline
\end{tabular}

$\varrho_{\mathrm{CH}}=$ Densidad $\left(\mathrm{kg} \mathrm{m}^{-3}\right) ; \mathrm{MOE}=$ Módulo de elasticidad $\left(\mathrm{MN} \mathrm{m}^{-2}\right) ; \mathrm{R}=$ Retención de sales de boro $\left(\mathrm{kg} \mathrm{m}^{-3}\right)$; $\overline{\mathrm{x}}=$ media; $\sigma=$ desviación estándar; $\mathrm{CV}=$ coeficiente de variación.

Los valores de la retención en la madera de G. ulmifolia, S. campanulata y A. religiosa (Tabla 1) fueron comparables con los resultados para maderas mexicanas. Sotomayor y Villaseñor (2016) reportan retenciones que van de 4,07 $\mathrm{kg} \mathrm{m}^{-3}$ a 14,18 $\mathrm{kg} \mathrm{m}^{-3}$. Las retenciones promedio en la madera de G. ulmifolia, S. campanulata y 
A.religiosa estuvieron en el intervalo reportado por Berrocal et al. (2004); Dhamodaran y Gnanaharan (2007); Simsek et al. (2010) y Tondi et al. (2012) quienes obtuvieron retenciones que van de $0,32 \mathrm{~kg} \mathrm{~m}^{-33}$ a $17,42 \mathrm{~kg} \mathrm{~m}^{-3}$. Las retenciones de G. ulmifolia, S. campanulata y A. religiosa fueron mayores a las sugeridas por la Asociación Americana de Preservadores de Madera $\left(2,72 \mathrm{~kg} \mathrm{~m}^{-3}\right)$ para la madera empleada en elementos estructurales (American Wood-Preservers' Association, 2014).

La madera de G. ulmifolia, S. campanulata y A. religiosa retuvo sales de boro en una cantidad mayor al mínimo recomendado por la Asociación Americana de Preservadores de Madera (American Wood-Preservers' Association, 2014). Posiblemente, estas sales y el tratamiento baño caliente-frío son recomendables para proteger estas especies del deterioro ocasionado por agentes biológicos y factores físicos.

El tratamiento no modificó la densidad de G. ulmifolia. En contraste, sí influyó en la densidad de $S$. campanulata y de A. religiosa. Estos resultados no estuvieron relacionados con la magnitud de la retención en la madera de cada una de estas especies. Sin embargo, la densidad de G. ulmifolia, fue mayor comparativamente a las de S. campanulata y de A. religiosa. Cuantitativamente, los resultados de retención son comparables con los de Alfaro (2013). Aplicando un proceso de preservado por inmersión, el autor presenta valores de retención de sales de boro para nueve especies de maderas tropicales: Hymenaea courbaril 23,2 $\mathrm{kg} \mathrm{m}^{-3}$, Terminalia amazonia $12, .9 \mathrm{~kg} \mathrm{~m}^{-3}$, Hieronyma alchorneoides $8,1 \mathrm{~kg} \mathrm{~m}^{-3}$, Cedrela odorata $5,7 \mathrm{~kg} \mathrm{~m}^{-3}$, Tectona grandis $9,1 \mathrm{~kg} \mathrm{~m}^{-3}$, Cupressus lusitanica 14,9 $\mathrm{kg} \mathrm{m}^{-3}$, Enterolobium cyclocarpum $7 \mathrm{~kg} \mathrm{~m}^{-3}$, Calophyllum brasiliense $15 \mathrm{~kg} \mathrm{~m}^{-3}$ y Gmelina arborea $8,7 \mathrm{~kg} \mathrm{~m}^{-3}$.

Para G. ulmifolia, S. campanulata y A. religiosa el análisis estadístico entre los módulos de elasticidad "antes tratamiento" y "después tratamiento" no indicó diferencias significativas. Este argumento permite inferir que el tratamiento de sales de boro no influyó en el módulo de elasticidad de la madera. Sin embargo, el cálculo aritmético de sus valores promedio (tabla 1) resultó en incrementos del 3,8 \% para G. ulmifolia y del 7,9\% para S. campanulata. En contraste, los módulos de elasticidad de A. religiosa "antes tratamiento" y "después tratamiento" permanecieron prácticamente iguales, coincidiendo este resultado con el de las pruebas estadísticas.

La cantidad de retención de sales de boro fue inversamente proporcional al módulo de elasticidad de la madera para las tres especies estudiadas. Comparativamente, S. campanulata retuvo $96 \%$ más sales de boro que A. religiosa y $48 \%$ más que G. ulmifolia. Este resultado es proporcional a la magnitud de los módulos de elasticidad de las tres especies. Mientras más grande es el módulo de elasticidad, la retención es menor. Este resultado se muestra en la figura 3, la cual presenta las regresiones entre el módulo de elasticidad (MOE) y la retención (R) para antes tratamiento (at) y después tratamiento (dt).

Este resultado es particular a las especies estudiadas y a las variables del tratamiento aplicado en este caso de estudio. Para apoyar este argumento, se puede citar a Simsek et al. (2013) quienes impregnaron madera de Fagus orientalis y Pinus sylvestris con una solución de sales de boro con una concentración al $4 \%$ y lograron retenciones de $21,1 \%$ para Fagus orientalis y de 28,8 \% para Pinus sylvestris. Los autores encontraron que el módulo de elasticidad disminuyó 10,57 \% para Fagus orientalis y 4,39 \% para 
Pinus sylvestris. Por su parte, Percin, Sofuoglu y Uzun (2015) impregnaron madera de Quercus petraea con sales de boro a una concentración de retención de $5 \%$, y lograron una retención de entre $6,73 \mathrm{~kg} \mathrm{~m}-3$ y 8,27 kg m-3. Los investigadores encontraron que el módulo de elasticidad disminuye entre $0,57 \%$ y $7,23 \%$ por el efecto del tratamiento con sales de boro.

Las diferencias en los resultados entre las investigaciones anteriores indican que cada procedimiento debe estar referido a las variables de referencia de las condiciones de ensayo, particularmente a la especie y la concentración de los reactivos químicos. Una vez contando con datos estadísticamente representativos, se pueden proponer procesos de impregnación a nivel industrial (Gérardin, 2016).

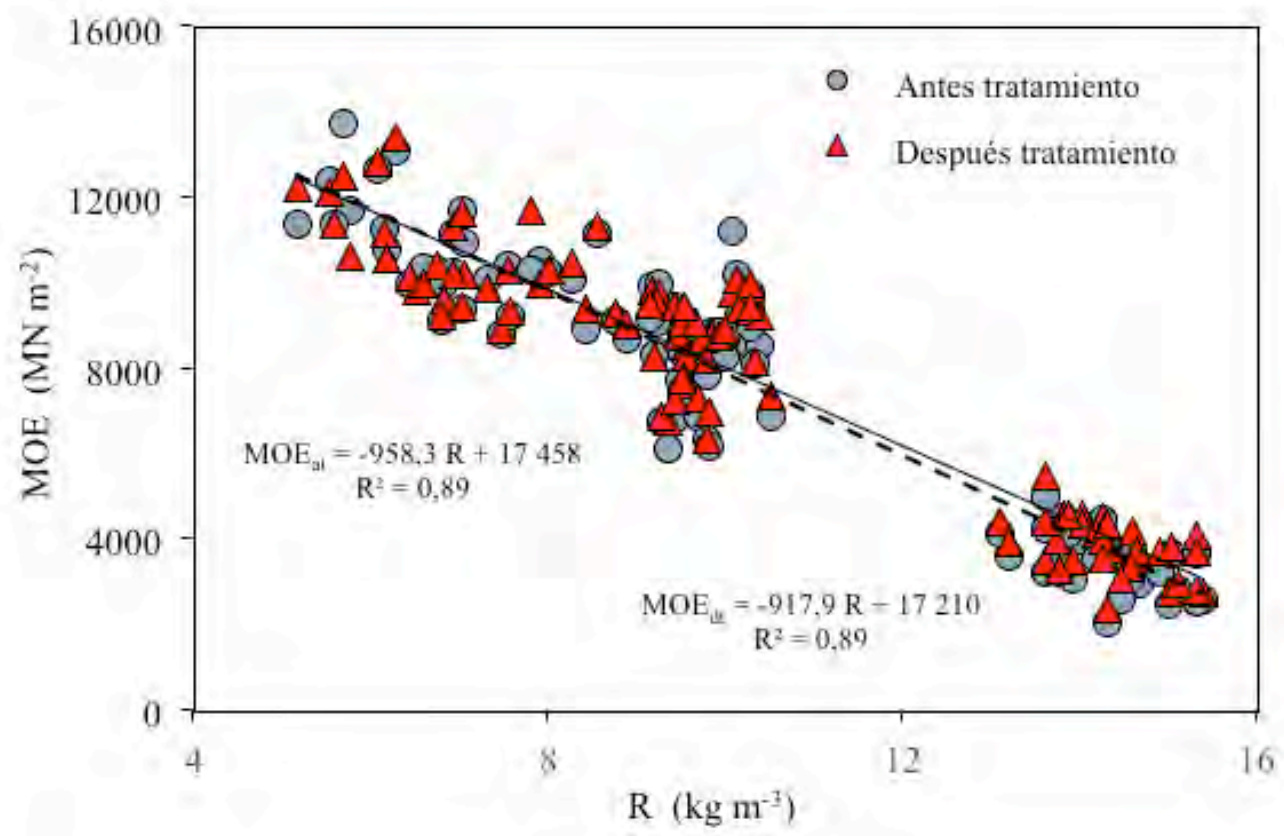

Figura 3. Regresiones entre el módulo de elasticidad (MOE) y la retención (R) para antes tratamiento (at) y después tratamiento $(\mathrm{dt})$.

\section{CONCLUSIONES}

El baño caliente-frío fue eficiente para impregnar con sales de boro madera de G. ulmifolia, S. campanulata y A. religiosa. Las maderas retuvieron cantidades diferentes de sales de boro aplicadas en baño caliente-frío. La densidad y el módulo de elasticidad de G. ulmifolia, S. campanulata y A. religiosa se comportan de manera diferente al tratamiento de impregnación. Los resultados confirman el paradigma propuesto por Shmulsky y Jones (2011) que rige en investigación e ingeniería de la madera: la caracterización del comportamiento mecánico de la madera requiere experimentar caso por 
caso cada especie en particular y cada procedimiento debe estar referido a las variables de referencia de las condiciones de ensayo. Una vez teniendo observaciones estadísticamente representativas, se pueden proponer tendencias en el comportamiento general para una especie en específico, y/o por agrupamiento de varias de ellas que denoten una tendencia similar. No obstante, para fines de diseño y cálculo ingenieril, es recomendable considerar el alcance de los resultados de este caso de estudio que se limita a madera de G. ulmifolia, S. campanulata y A. religiosa.

\section{AGRADECIMIENTOS}

La investigación estuvo patrocinada por la Universidad Michoacana de San Nicolás de Hidalgo, México y por el Institut Català de la Fusta del Centre Tecnològic Forestal de Catalunya, España.

\section{REFERENCIAS}

Alfaro P. J. D. (2013). Estudio de retención y penetración de tres preservantes comerciales en nueve especies maderables presentes en Costa Rica. Ingeniería, 23(1), 107-119.

American Wood-Preservers' Association. (2014). P5-07 Standard for Waterborne Preservatives. Chicago: American Wood-Preservers' Association.

Ávila C., L.E. A., Herrera F., M. A. y Raya, G., D. (2012). Preservación de la Madera en México. Morelia: Universidad Michoacana de San Nicolás de Hidalgo.

Baysal, E., Sonmez, A., Colak, M. y Toker, H. (2006). Amount of leachant and water absorption levels of wood treated with borates and water repellents. Bioresource Technology, 97(18), 2271- 2279.

Berrocal, A., Muñoz, F. y González, G. (2004). Ensayo de penetrabilidad de dos preservantes a base de boro en madera de melina (Gmelina arborea) crecida en Costa Rica. Revista Forestal Mesoamericana Kurú, 1(3), 1-12.

Bettiol, G., Ceccato, F., Pigouni, A. E., Modena, C. y Simonini, P. (2016). Effect on the Structure in Elevation of Wood Deterioration on Small-Pile Foundation: Numerical Analyses. International Journal of Architectural Heritage, 10(1), 44-54.

Caldeira, F. (2010). Boron in Wood Preservation. A Review in its Physico-Chemical Aspects. Silva Lusitana, 18(2), 179-196.

Cruz D., J. (2010). Manual para la Protección contra el Deterioro de la Madera. México: Comisión Nacional Forestal.

Dhamodaran, K. y Gnanaharan, R. (2007). Boron impregnation treatment of Eucalyptus grandis Wood. Bioresource Technology, 98(11), 2240-2242.

Gérardin, P. (2016). New alternatives for wood preservation based on thermal and chemical modification of wood. A review. Annals of Forest Science, 73(3), 559-570.

International Organization for Standardization. (2014a). ISO 13061-1:2014. Wood. Determination of moisture content for physical and mechanical tests. Geneva: International Organization for Standardization. 
International Organization for Standardization. (2014b). ISO 13061-2:2014. Wood. Determination of density for physical and mechanical tests. Geneva: International Organization for Standardization.

Koman, S., Feher, S., Abraham, J., y Taschner, R. (2013). Effect of knots on the bending strength and the modulus of elasticity of wood. Wood Research, 58(4), 617-626.

Lloyd, J. D., Fogel, J. L. y Vizel, A. (2001). The use of Zirconium as an inert fixative for borates in Preservation. Stockholm: International Research Group on Wood Protection.

Obanda, D. D., Shupe, T. F. y Barnes, H. M. (2008). Reducing leaching of boron-based wood preservatives. A review of research. Bioresource Technology, 99(15), 7312-7322.

Obounou-Akong, F., Gérardin, P., Thévenon, M. F. y Gérardin-Charbonnier, C. (2015). Hydrogel-based boron salt formulations for wood preservation. Wood Science and Technology, 49(3), 443-456.

Organismo Nacional de Normalización y Certificación de la Construcción y Edificación. (1999). NMX-C-410-ONNCCE-1999. Retención y Penetración de Sustancias Preservadoras en Madera. Métodos de Prueba. México: Organismo Nacional de Normalización y Certificación de la Construcción y Edificación.

Percin, O., Sofuoglu, S. D. y Uzun, O. (2015). Effects of Boron Impregnation and Heat Treatment on Some Mechanical Properties of Oak (Quercus petraea Liebl.) Wood. BioResources, 10(3), 3963-3978.

Salman, S., Pétrissans, A., Thévenon, M. F., Dumarcay, S., Perrin, D., Pollier, B. y Gérardin. P. (2014). Development of new wood treatments combining boron impregnation and thermo modification: effect of additives on boron leachability. European Journal of Wood Products, 72(3), 355-365.

Shmulsky, R. y Jones, P. D. (2011). Forest Products and Wood Science. Hoboken: Wiley.

Simsek, H., Baysal, E. y Peker, H. (2010). Some mechanical properties and decay resistance of wood impregnated with environmentally friendly borates. Construction and Building Materials, 24(11), 2279-2284.

Simsek, H., Baysal, E., Yilmaz, M., y Culha, F. (2013). Some mechanical properties of wood impregnated with environmentally-friendly boron and copper based chemicals. Wood Research, 58(3), 495-504.

Sotomayor C., J. R. (2015). Banco FITECMA de Características Físico-Mecánicas de Maderas Mexicanas. Morelia: Universidad Michoacana de San Nicolás de Hidalgo.

Sotomayor C., J. R. y Ramírez P., M. (2013). Densidad y características higroscópicas de maderas mexicanas. Base de datos y criterios de clasificación. Investigación e Ingeniería de la Madera, 9(3), 3-29.

Sotomayor C., J. R. y Villaseñor A., J. M. (2016). Retención y absorción de solución de sales boro de diez maderas mexicanas. Acta Universitaria, 26(2), 18-25.

Temiz, A., Alfredsen, G., Eikenes, M. y Terziev, N. (2008). Decay resistance of wood treated with boric acid and tall oil derivates. Bioresource Technology, 99(7), 2102- 2106.

Tondi, G., Wieland, S., Lemenager, N., Petutschnigg, A., Pizzi, A. y Thevenon, M. F. (2012). Efficacy of tannin in fixing boron in wood: fungal and termite resistance. BioResources, $7(1), 1238-1252$. 
Tsunoda, K. (2001). Preservatives properties of vapor-boron-treated wood and wood based composites. Journal of Wood Science, 47(2), 149-53.

Walker, J. C. F. (2006). Primary Wood Processing. Principles and Practice. Dordrecht: Springer.

Zauer, M., Pfriem, A. y Wagenführ, A. (2013). Toward improved understanding of the cell-wall density and porosity of wood determined by gas pycnometry. Wood Science and Technology, 47(8), 1197-1211.

Zauer, M., Hempel, S., Pfriem, A., Mechtcherine, V. y Wagenführ, A. (2014). Investigations of the pore-size distribution of wood in the dry and wet state by means of mercury intrusion porosimetry. Wood Science and Technology, 48(6), 1229-1240. 